\title{
Enhancing Routing Protocol for WIRELESS SENSOR NETWORK TO ADVANCE NETWORK LIFETIME
}

\author{
Mohammad Abdus Salam ${ }^{1}$, Kosta Varnavas ${ }^{2}$ and Bhargava Maddireddy ${ }^{1}$ \\ ${ }^{1}$ Department of Computer Science, Southern University, Baton Rouge, Louisiana, USA \\ ${ }^{2}$ NASA Marshall Space Flight Center, Huntsville, USA
}

\begin{abstract}
In wireless sensor networks, nodes are operated by batteries. To keep the network alive for a long time, it is crucial that we maintain battery life. There are many proposals on how to preserve the battery life. In this research, we focus on the routing protocols to prolong the network lifetime. Our proposed protocol is based on two basic protocols, namely, LEACH (Low-Energy Adaptive Clustering Hierarchy) and PEGASIS (Power Efficient Gathering in Sensor Information System). The proposed method compared favorably with these two basic protocols and our previously proposed cluster-based protocol in terms of network lifetime.
\end{abstract}

\section{KEYWORDS}

Sensor network, routing protocol, network lifetime, and wireless communication

\section{INTRODUCTION}

The application of wireless sensor networks is enormous. In our daily life, we interact with sensors on many occasions. These sensors are deployed in security and surveillance systems, environmental monitoring, industries, precision agriculture, disaster response, automotive vehicle, military, spacecraft, underwater sensor networks, and many more places [1], [2]. Wireless sensor networks are composed of many sensor nodes that have sensing, computing, and communicating capabilities [3]. These nodes collaborate among themselves to sense and collect crucial data such as audio, video, seismic, or others as necessary. After collecting the data, these nodes perform computation and finally transmit data to the neighboring nodes or directly to the base station depending upon the routing protocol. The number of sensor nodes for each application varies ranging from tens to hundreds or even thousands depending upon the application and the size of the network. These wireless sensor nodes are powered by batteries and they are energy constrained. Once the battery of a sensor node is dead or below a certain threshold value, it is hard to replace it, and therefore, the node is considered as a dead node. There are many research proposals on how to prolong battery life, i.e. the network lifetime by using the routing protocols [4]. An energy-efficient routing protocol can improve the lifetime of a network and therefore the degree of network performance [5]. It is necessary to design a wireless communication protocol that will maximize the node's lifetime and minimize the node failure by collaborating with neighboring nodes [6], [7].

Many researchers around the globe have approached in various ways to improve the network lifetime and energy efficiency of the wireless sensor networks. In [8], hybrid features namely 
node residual energy and node proximity are used to determine the cluster head to increase network lifetime. Whereas, Mirza and Garimella [9] have divided the sensor field into different levels and introduced sectoring and clustering in those levels to increase network lifetime. Huang et al. [10] have proposed an annulus sector grid aided routing protocol called ASGRP to improve energy efficiency and prolong the network lifetime of wireless sensor networks. Arjunan and Sujatha [11] have used two separate techniques to handle clustering and routing. They have used fuzzy logic to select cluster and ant colony mechanism for routing of data to eliminate hot spot problems and extend network lifetime. Darabkh et al. [12] proposed a new algorithm for clustering and routing of sensor field called balanced power-aware clustering and routing protocol (BPA-CRP) to improve load balancing and energy awareness by dividing the network into equal-sized layers and clusters.

In this paper, we have proposed a new wireless communication protocol which is based on two basic protocols, namely, LEACH and PEGASIS. Earlier [13], we have proposed cluster-based routing protocol which was also based on these two basic protocols - LEACH and PEGASIS. In this paper, we proposed hierarchy-based routing protocol that outperforms our previously proposed cluster-based protocol as well as LEACH and PEGASIS protocols. In the following sections, we will briefly explain the two basic protocols, previously proposed cluster-based protocol, and provide detail of the newly proposed protocol. In the end, we will compare the performance of these protocols.

\section{RELATED WORK}

In this section, we will provide a brief description of three protocols: LEACH, PEGASIS, and previously proposed cluster-based protocol.

\subsection{LEACH Protocol}

The LEACH (Low-Energy Adaptive Clustering Hierarchy) protocol was developed at the MIT Lab by Heinzelman et al. [14]. It is a self-organizing and adaptive clustering hierarchy protocol. The operation of LEACH is done in two phases: the set-up phase and the steady-state phase. The cluster formation is done during the set-up phase. Depending upon the node's energy level and the number of times it serves as a cluster head $(\mathrm{CH})$, a node is chosen as a cluster head. Once a node is decided as a cluster head, it broadcasts joining request messages to its neighboring nodes. If the neighboring nodes receive multiple joining requests, it will join the one with a stronger signal. The member nodes must inform the cluster head that they have joined in that cluster. Once a cluster is formed, the cluster head node creates a TDMA (time division multiple access) schedules among the member nodes to receive data. In the steady-state phase, member nodes transmit the sensed data to the cluster head node. Once the cluster head node receives all the sensed data from its member nodes, it conducts signal processing to compress the received data and transmits to the base station (BS).

Figure 1 shows how the LEACH protocol works. The basic protocol was simulated with 100 sensor nodes and five clusters. The nodes marked with the same symbol belong to the same cluster and the cluster heads are marked with a filled dark circle. These clusters are dynamic, i.e. the cluster and cluster head change after each round. The number of clusters in a network depends upon the environments, size of the network, and other network parameters. The base station is fixed and located far from sensor nodes. These nodes are considered homogeneous and energy constrained. 


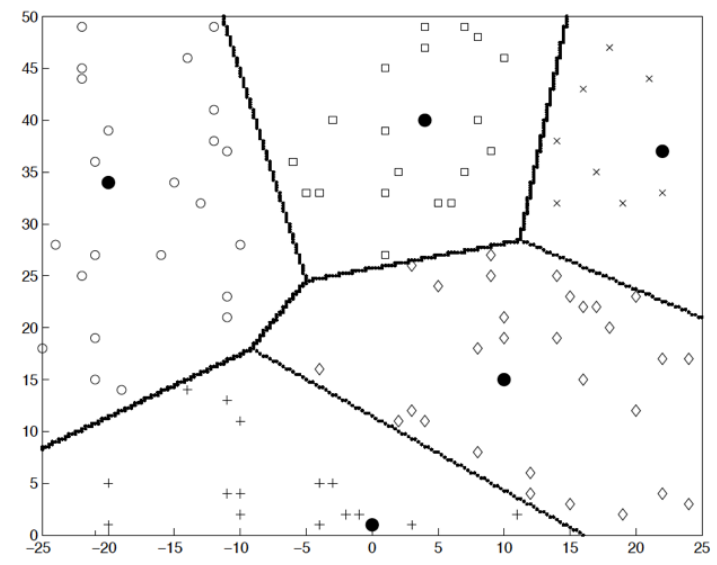

Figure. 1. LEACH protocol operation [9]

The main drawback of LEACH is that the cluster head node transmits data directly to the base station and it causes a lot of energy consumption if the base station is far from that cluster head. There are several modified version of LEACH protocols available in the market such as LEACHC (centralized) [15], Energy-LEACH [16], multihop-LEACH [16], LEACH-B (balanced) [17] and many others [18]. Al-Baz and El-Sayed have proposed another modified version of LEACH called node ranked LEACH to improve network performance in terms of energy consumption and network lifetime [19].

\subsection{PEGASIS Protocol}

The PEGASIS (Power Efficient Gathering in Sensor Information Systems) protocol was proposed right after the LEACH protocol at the Aerospace Corporation Lab by Lindsey and Raghavendra [20].

In PEGASIS, a greedy algorithm is used to form the chain among the sensor nodes assuming that the base station has the global knowledge of the entire network. The chain formation starts from the furthest node from the base station. This initial node (i.e. the furthest node from the base station) will connect to the nearest neighbor node and that nearest neighbor node will connect to another nearest neighbor node and so on until all nodes are connected in the chain based on a greedy algorithm. The node closest to the base station (BS) will be a leader node who will be responsible to transmit data to the base station. Once a node dies, the chain is reconstructed in the same manner excluding the dead node. Each node performs data fusion and transmits data to the nearest neighbor node to whom it is connected. Eventually, all the sensed information comes to the leader node and it transmits to the base station as explained in Figure 2.

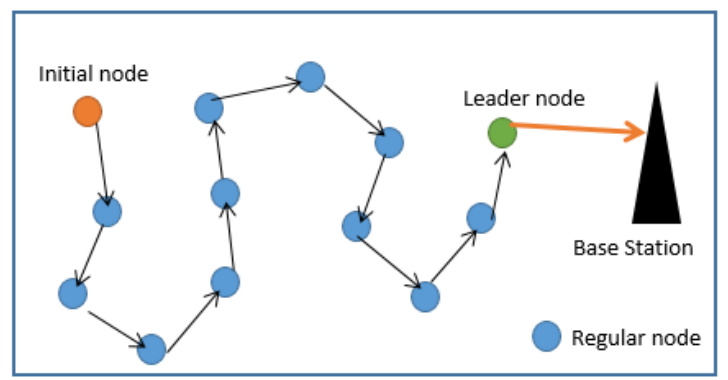

Figure 2. PEGASIS protocol architecture 
The PEGASIS protocol outperforms the LEACH protocol by approximately $2 \mathrm{x}$ the number of rounds when $1 \%, 20 \%, 50 \%$, and $100 \%$ of nodes die for a $50 \mathrm{~m} \times 50 \mathrm{~m}$ network [17]. Since the publication of PEGASIS, there have been many modified versions of PEGASIS available by many scientists around the globe. Li et al. [21] have proposed an ant colony-based algorithm instead of the greedy algorithm to form the chain. Feng et al. have proposed another version of PEGASIS [22] that assigns each node weight and uses a weighting mechanism to select the transmitter node.

\subsection{Cluster-Based Proposed Protocol}

Our previously proposed protocol was based on LEACH and PEGASIS protocols [13]. In that protocol, we assume that the base station (BS) has the knowledge of the sensor nodes' physical locations and it calculates the distances from the base station to all other nodes. It forms clusters among the sensor nodes based upon the principle of LEACH protocol and in each cluster, the node nearest to the base station serves as a cluster head $(\mathrm{CH})$ or leader node. Since the base station has the knowledge of each sensor node, the furthest node from the base station in each cluster will be the initial node to start the transmission to the nearest node in the chain of a specific cluster as per the principle of PEGASIS protocol. Once the chain formation is done in each cluster, the base station will calculate the distances of each cluster head and the cluster head furthest from the base station will be the initial node to start transmission to the nearest cluster head node in the chain.

Figure 3 explains the chain formation and data transmission of the previously proposed clusterbased protocol. Here we divided the nodes into five clusters with cluster heads $\mathrm{CH} 1, \mathrm{CH} 2, \mathrm{CH} 3$, $\mathrm{CH} 4$, and $\mathrm{CH} 5$. Each cluster has an initial node and a cluster head node. Chain formation starts at the initial node and ends at the cluster head node. Among the cluster heads $(\mathrm{CH} 1, \mathrm{CH} 2, \mathrm{CH} 3$, $\mathrm{CH} 4$, and $\mathrm{CH} 5)$, the furthest cluster head (CH1) node from the base station becomes the initial node and the nearest cluster head (CH5) node from the base station becomes the final or leader node to transmits data to the base station.

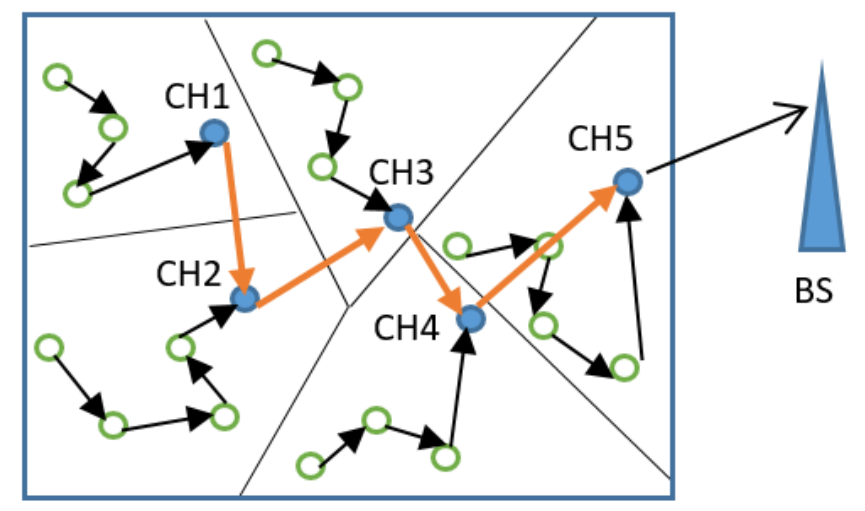

Figure 3. Cluster-based proposed protocol architecture

\section{Hierarchy-BASEd Proposed Protocol}

Our proposed protocol is based on two basic protocols: LEACH and PEGASIS. The proposed routing protocol combined the features of hierarchy and cluster. In the following sections, we will discuss the architecture and performance evaluation of the proposed protocol. 


\subsection{Architecture of the Proposed Protocol}

The proposed hierarchy-based protocol architecture is shown in Figure 4. The white circles represent member nodes and the black circles represent cluster head $(\mathrm{CH})$ of a cluster. In this proposed algorithm, it is assumed that the base station (BS) has the knowledge of the physical location of each sensor node and BS calculates the distances of all the sensor nodes. The nodes are categorized into different levels based upon their distances from the BS. In this proposed architecture, the entire network is divided into three hierarchical levels and sensor nodes into each level form clusters among themselves. The cluster formation is performed based on the LEACH algorithm. As in LEACH, in each cluster, there is a cluster head and the cluster head is chosen depending on the distance from the base station (BS) and energy level. The chain formation among the nodes in a cluster and among the cluster heads of various clusters is done based on the PEGASIS algorithm.

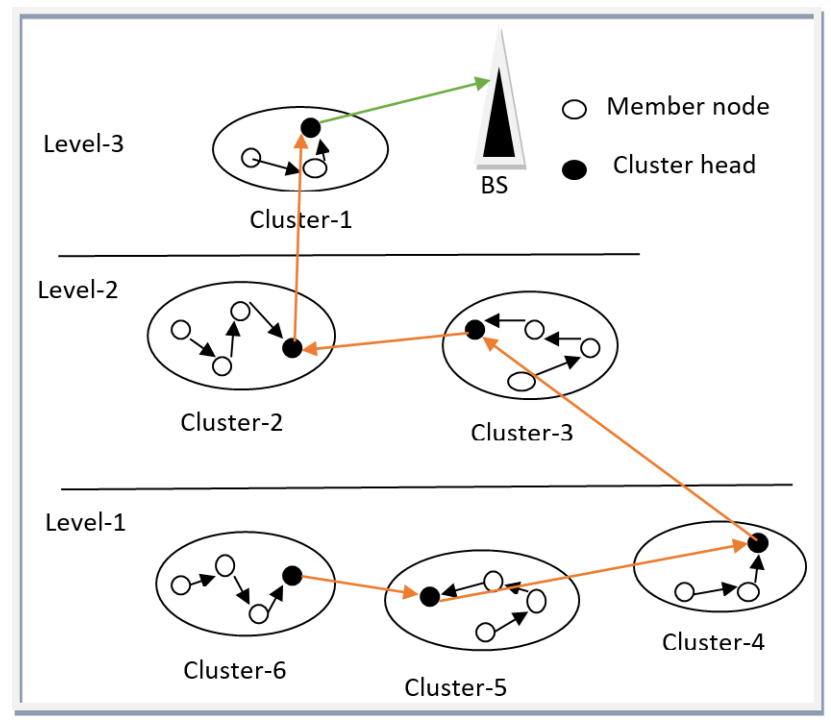

Figure 4. Proposed hierarchy-based protocol architecture

The working principle of the proposed architecture is described in the following steps.

Step-1: The BS calculates the distances of all the nodes of the entire network.

Step-2: The nodes are categorized into various levels based on their distances from the base station.

Step-3: In each level, cluster head selection and cluster formation are done based on the working principle of the LEACH algorithm.

Step-4: In each cluster, the chain formation is done based on the PEGASIS algorithm. The furthest node from the BS will be the initial node and the cluster head will be the final or leader node of that cluster.

Step-5: Among all the cluster heads in the same level, the furthest distance $\mathrm{CH}$ from the base station will be the initial data transmission node and the cluster head nearest to the base station will be the final node. This final node will transmit data to the next level initial node which is also chosen in the same manner.

Step-6: Finally, the last cluster head in the last level will send all the data from the entire network to the base station.

In Figure 4, there are six clusters; three in level-1, two in level-2, and one in level-3. After the formation of chains in each level and each cluster, i.e. among the six clusters, the cluster head in 
cluster-6 initiates the data transmission (since it is the furthest $\mathrm{CH}$ from the base station) and it sends data to cluster head 4 through cluster head 5. The cluster head 4 transmits data to the nearest upper-level cluster head node which is in cluster 3. After receiving the data from the $\mathrm{CH}$ in cluster 3, the cluster head in cluster 2 transmits it to the cluster head in cluster 1. Finally, the cluster head in cluster 1 transmits the entire network data to the base station.

\subsection{Simulation of the Proposed Protocol}

In our simulation, we have considered 60 sensor nodes to analyze network performance. Java program is coded according to the proposed algorithm. The base station is located at $(100,100)$. Initially, all the nodes in the network will have the same energy of 1 Joule per node. In each round of the transmission, each node will transmit a 2000-bit data packet to the base station. When the energy dissipation of a particular node goes below the threshold value, it is considered as a dead node. The following Figures (5), (6), and (7) show the status of the nodes at their different rounds. The green color represents an alive node and the red color represents a dead node. The star at the upper right corner at the location of $x=100$ and $y=100$ represents the base station.

Figure 5 displays the initial set up and (x, y) coordinates of all the 60 sensor nodes. It is assumed that all the sensor nodes have the same amount of energy to start with. They are all alive at this round of the network.

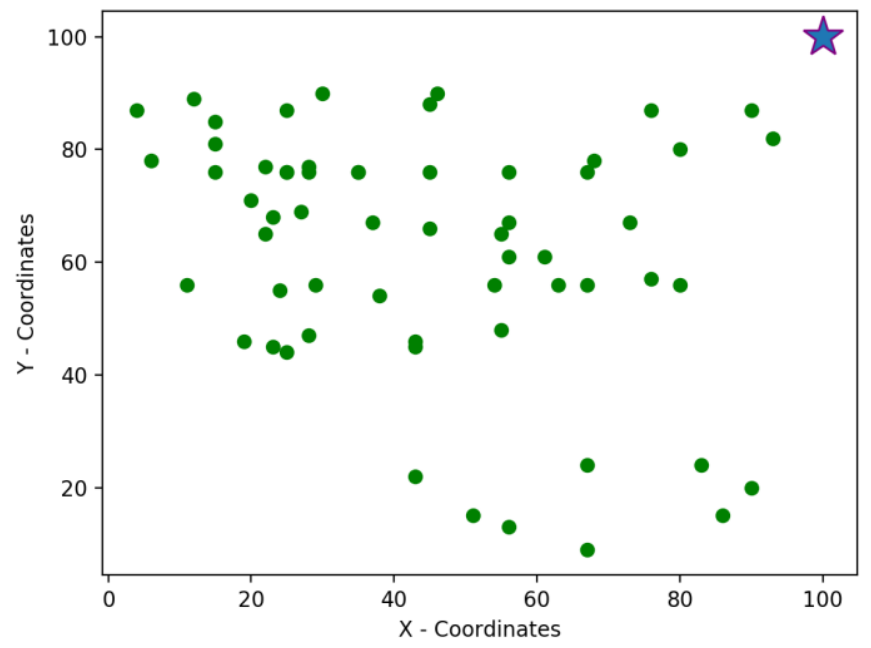

Figure 5. Initial setup of sensor nodes for the proposed protocol.

After the $10^{\text {th }}$ round, we see only six nodes are dead and most of them are alive (Figure 6). Unlike the minimum transmission energy (MTE) protocol or direct transmission protocol as described in [9], [10], where the initial energy dissipation is concentrated either closest areas to the base station or furthest areas from the base station, respectively. In MTE protocol, the closer nodes are being used by the further nodes as a router to transmit data to the base station in the chain which causes the nearest nodes to die earlier. Whereas, in the direct transmission protocol, each node transmits directly to the base station which causes the further nodes to die faster compared to the nearer nodes. In either of these cases, areas, where sensors are dead become unmonitored. In the proposed method, we have noticed that dead nodes are not concentrated in one single area rather it is distributed among various areas and we still are able to monitor the field of deployment without any disruption. 


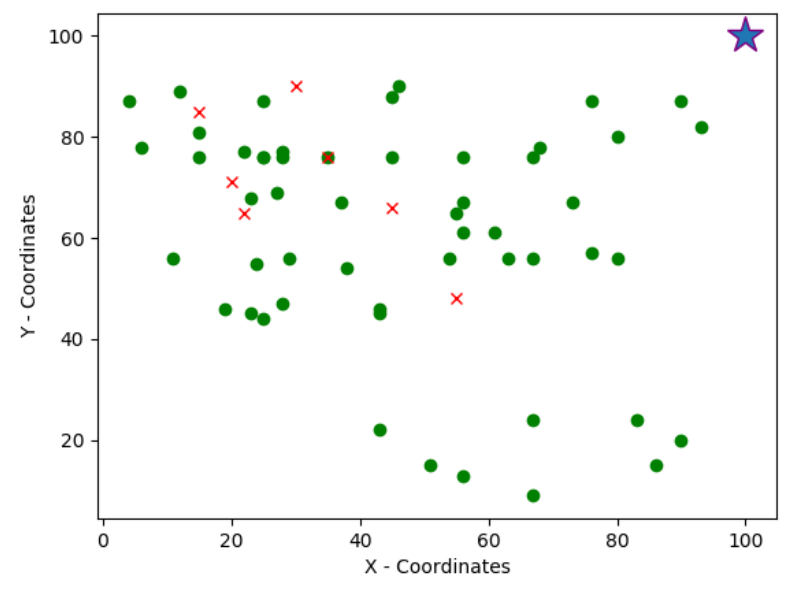

Figure 6. Nodes status after $10^{\text {th }}$ round for the proposed protocol.

Figure 7 shows the various nodes status after the $30^{\text {th }}$ round. Here we notice that the number of survival nodes is more compared to other protocols. Moreover, the alive nodes are not concentrated in one single area rather it is distributed almost all over the deployed areas which makes this protocol more promising compared to other protocols.

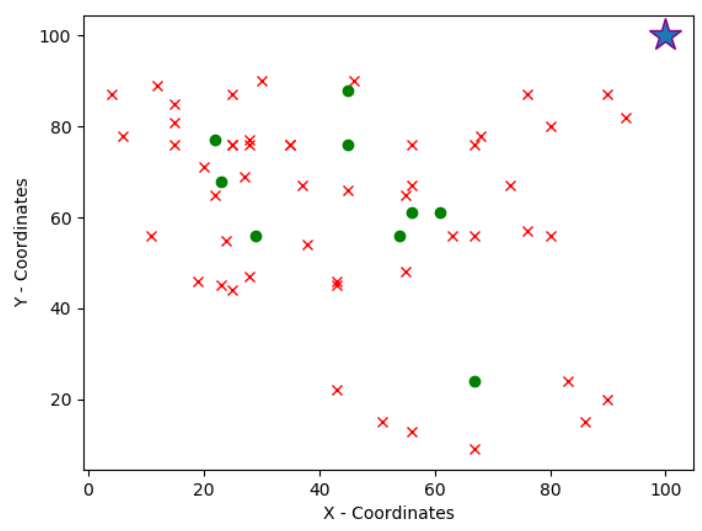

Figure 7 . Nodes status after $30^{\text {th }}$ round for the proposed protocol.

The survival status of different nodes is summarized in Figure 8. We can deduct from this figure which node is disappearing at what round. For example, node number 60 dies at round 10 and node number 2 dies in round 15 .

The complete list of all the 60 sensor nodes is provided in Table 1 . The first column shows the 60 sensor nodes in sequence starting from node number 1 through node number 60 . The second column displays the $\mathrm{x}$ and $\mathrm{y}$ coordinate of a sensor node. For example, the $(\mathrm{x}, \mathrm{y})$ coordinate of node 20 is $(56,61)$ and the $(x, y)$ coordinate of node 40 is $(20,71)$. 


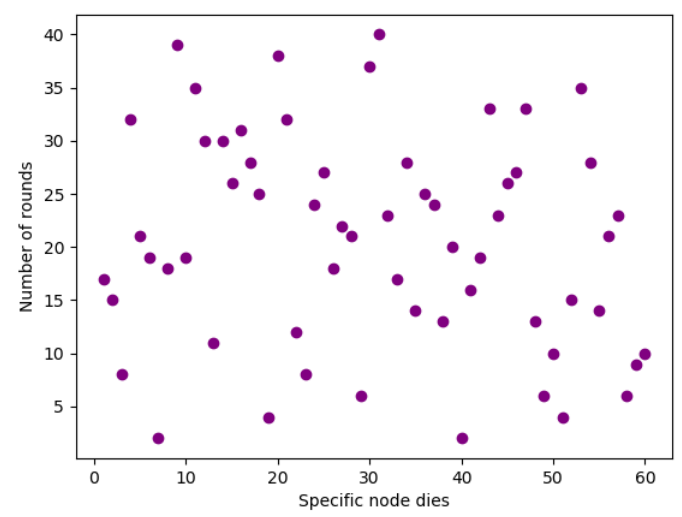

Figure 8. Nodes' survival status.

The third column represents the distances of each node from the base station. As we know that the distance between two coordinates points $\mathrm{P}\left(\mathrm{x}_{1}, \mathrm{y}_{1}\right)$ and $\mathrm{Q}\left(\mathrm{x}_{2}, \mathrm{y}_{2}\right)$ can be calculated as:

$$
\text { distance }=\mathrm{F}\left[\left(\mathrm{x}_{2}-\mathrm{x}_{1}\right)^{2}+\left(\mathrm{y}_{2}-\mathrm{y}_{1}\right)^{2}\right]
$$

The base station is located at $(100,100)$. The distance from node 10 to the base station is 42.6 and the distance from node 50 to the base station is 70.7. For example, node number 3 is located at $\left(\mathrm{x}_{3}, \mathrm{y}_{3}\right)=(15,85)$ and the base station which is located at $(\mathrm{x}, \mathrm{y})=(100,100)$ can be calculated as:

$$
\mathrm{d}=\mathrm{W}\left[\left(\mathrm{x}-\mathrm{x}_{3}\right)^{2}+\left(\mathrm{y}-\mathrm{y}_{3}\right)^{2}\right]=\mathrm{V}_{\mathrm{V}}\left[(100-15)^{2}+(100-85)^{2}\right]=86.3 .
$$

In all of these three protocols (PEGASIS, proposed cluster and proposed hierarchy), we kept the sensor nodes at the same physical location, i.e. these sensor nodes are static.

Table 1. Node's location, distance, and the round at which a specific node dies for the three protocols

\begin{tabular}{|c|c|c|c|c|c|}
\hline $\begin{array}{c}\text { Sensor } \\
\text { nodes }\end{array}$ & $\begin{array}{c}\text { Location } \\
(\mathrm{x}, \mathrm{y})\end{array}$ & $\begin{array}{c}\text { Distance } \\
\text { from BS } \\
(\text { meter })\end{array}$ & PEGASIS & $\begin{array}{c}\text { Proposed } \\
\text { Cluster }\end{array}$ & $\begin{array}{c}\text { Proposed } \\
\text { Hierarchy }\end{array}$ \\
\hline 1 & 76,14 & 89.3 & 12 & 26 & 17 \\
\hline 2 & 12,89 & 88.7 & 15 & 25 & 15 \\
\hline 3 & 15,85 & 86.3 & 20 & 24 & 8 \\
\hline 4 & 67,24 & 82.9 & 3 & 20 & 32 \\
\hline 5 & 80,56 & 48.3 & 2 & 5 & 21 \\
\hline 6 & 25,44 & 93.6 & 3 & 28 & 19 \\
\hline 7 & 45,66 & 64.7 & 8 & 13 & 2 \\
\hline 8 & 28,47 & 89.4 & 25 & 27 & 18 \\
\hline 9 & 45,76 & 60 & 7 & 12 & 39 \\
\hline 10 & 73,67 & 42.6 & 5 & 3 & 19 \\
\hline 11 & 29,56 & 83.5 & 25 & 21 & 35 \\
\hline 12 & 27,69 & 79.3 & 25 & 17 & 30 \\
\hline 13 & 37,67 & 71.1 & 3 & 14 & 11 \\
\hline 14 & 56,67 & 55 & 4 & 9 & 30 \\
\hline 15 & 46,90 & 54.9 & 2 & 7 & 26 \\
\hline 16 & 22,77 & 81.3 & 5 & 18 & 31 \\
\hline
\end{tabular}


International Journal of Wireless \& Mobile Networks (IJWMN) Vol. 12, No. 3, June 2020

\begin{tabular}{|c|c|c|c|c|c|}
\hline 17 & 11,56 & 99.3 & 9 & 18 & 28 \\
\hline 18 & 25,76 & 78.7 & 20 & 16 & 25 \\
\hline 19 & 22,65 & 85.5 & 15 & 23 & 4 \\
\hline 20 & 56,61 & 58.8 & 7 & 11 & 38 \\
\hline 21 & 61,61 & 55.2 & 20 & 14 & 32 \\
\hline 22 & 24,55 & 88.3 & 4 & 26 & 12 \\
\hline 23 & 35,76 & 69.3 & 4 & 15 & 8 \\
\hline 24 & 4,87 & 96.9 & 25 & 29 & 24 \\
\hline 25 & 51,15 & 98.1 & 12 & 31 & 27 \\
\hline 26 & 67,76 & 40.8 & 5 & 8 & 18 \\
\hline 27 & 43,22 & 96.6 & 25 & 28 & 22 \\
\hline 28 & 83,24 & 77.9 & 10 & 20 & 21 \\
\hline 29 & 90,87 & 16.4 & 1 & 6 & 6 \\
\hline 30 & 63,56 & 57.5 & 13 & 15 & 37 \\
\hline 31 & 54,56 & 63.7 & 3 & 16 & 40 \\
\hline 32 & 43,46 & 78.5 & 12 & 15 & 23 \\
\hline 33 & 25,87 & 76.1 & 20 & 18 & 17 \\
\hline 34 & 67,56 & 55 & 25 & 12 & 28 \\
\hline 35 & 15,76 & 88.3 & 7 & 24 & 14 \\
\hline 36 & 19,46 & 97.3 & 15 & 30 & 25 \\
\hline 37 & 56,76 & 50.1 & 9 & 10 & 24 \\
\hline 38 & 76,87 & 27.3 & 2 & 7 & 13 \\
\hline 39 & 23,45 & 94.6 & 8 & 16 & 20 \\
\hline 40 & 20,71 & 85.1 & 6 & 22 & 2 \\
\hline 41 & 68,78 & 38.8 & 1 & 9 & 16 \\
\hline 42 & 38,54 & 77.2 & 8 & 22 & 19 \\
\hline 43 & 23,68 & 83.4 & 3 & 23 & 33 \\
\hline 44 & 67,9 & 96.8 & 20 & 31 & 23 \\
\hline 45 & 56,13 & 97.5 & 20 & 32 & 26 \\
\hline 46 & 25,76 & 78.7 & 4 & 24 & 27 \\
\hline 47 & 45,88 & 56.3 & 8 & 12 & 33 \\
\hline 48 & 28,77 & 75.6 & 2 & 20 & 13 \\
\hline 49 & 35,76 & 69.3 & 13 & 17 & 6 \\
\hline 50 & 30,90 & 70.7 & 6 & 18 & 10 \\
\hline 51 & 55,48 & 68.8 & 3 & 15 & 4 \\
\hline 52 & 28,76 & 75.9 & 17 & 21 & 15 \\
\hline 53 & 55,65 & 57 & 10 & 9 & 35 \\
\hline 54 & 43,45 & 79.2 & 13 & 26 & 28 \\
\hline 55 & 80,80 & 28.3 & 8 & 8 & 14 \\
\hline 56 & 6,78 & 96.5 & 5 & 11 & 21 \\
\hline 57 & 76,57 & 49.2 & 9 & 10 & 23 \\
\hline 58 & 86,15 & 86.1 & 12 & 28 & 6 \\
\hline 59 & 93,82 & 19.3 & 4 & 6 & 9 \\
\hline 60 & 15,81 & 87.1 & 10 & 29 & 10 \\
\hline
\end{tabular}

The fourth column demonstrates the lifetime of each sensor when using the PEGASIS algorithm. For example, node 49 dies in round 13 and node 59 dies in round 4.

The fifth column displays the lifetime of sensor nodes when we apply the proposed cluster-based algorithm. For example, node 7 dies in round 13 and node 57 dies in round 10. 
The sixth column shows the nodes' lifetime status for the proposed hierarchy-based protocol. For example, 46 dies in round 27 whereas node 54 dies in round 28.

If we look carefully at Table 1, we find that the survival status of the proposed hierarchy-based protocol is better compared to the PEGASIS and proposed cluster-based protocols. For example, node 31 dies in round 40 in case of proposed hierarchy-based protocol whereas the same node dies earlier in rounds 3 and 16 for PEGASIS and proposed cluster-based protocol, respectively. Only a few cases some of our nodes are dying earlier than PEGASIS and proposed cluster-based protocol due to their location and functionality. For example, node 40 dies in round 2 for the proposed hierarchy-based protocol whereas it dies in round 6 and 22 for PEGASIS and proposed cluster-based protocol, respectively.

Overall, the nodes live longer for the proposed hierarchy-based protocol compared to the other two protocols. This instance is shown in Figure 9. Here, it is showing the various percentage of nodes death at different rounds. For example, the survival rate of the first $10 \%$ nodes is better in the proposed cluster-based protocol compared to the proposed hierarchy-based protocol and PEGASIS. The proposed hierarchy-based protocol compared favorably after the death of 50\% nodes. The $100 \%$ disappearance of all the nodes occurs after approximately in round 26,32 , and 40 for PEGASIS, proposed cluster, and proposed hierarchy protocol, respectively.

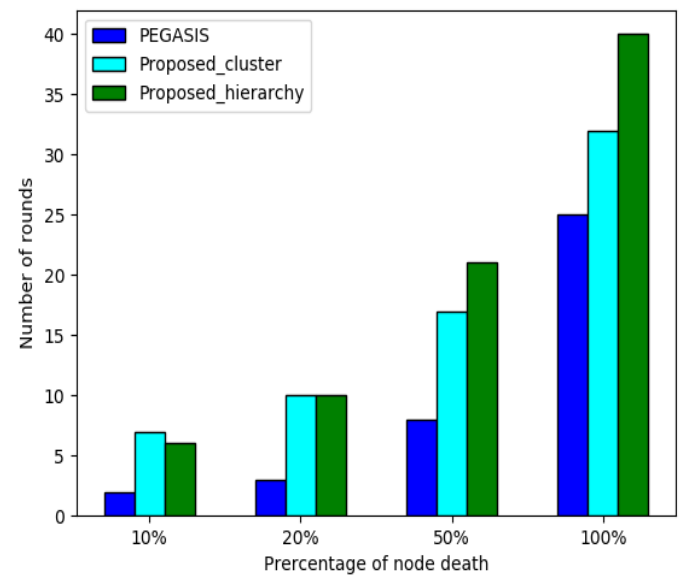

Figure 9. Performance results of PEGASIS, cluster based proposed protocol, and hierarchy based proposed protocol

Here we consider that the network lifetime of the wireless sensor network exists until the last surviving sensor node dies or the energy of the last sensor node goes below the threshold value to be considered as a dead node. Figure 10 compares the lifetime of these three protocols.

In PEAGASIS protocol, the nodes are started to die earlier, and the entire network became dead after the $26^{\text {th }}$ round of data transmission. Whereas, in the proposed cluster-based protocol, the entire network is alive until their $32^{\text {nd }}$ round. Among these three protocols, the proposed hierarchy-based protocol outperforms other protocols, it survives till the $40^{\text {th }}$ round of data transmission. Both of the proposed cluster-based and hierarchy-based algorithms extend the lifetime of the network compared to LEACH and PEGASIS. Since PEGASIS outperforms $\mathrm{LEACH}$, we have not compared here with the LEACH protocol. 


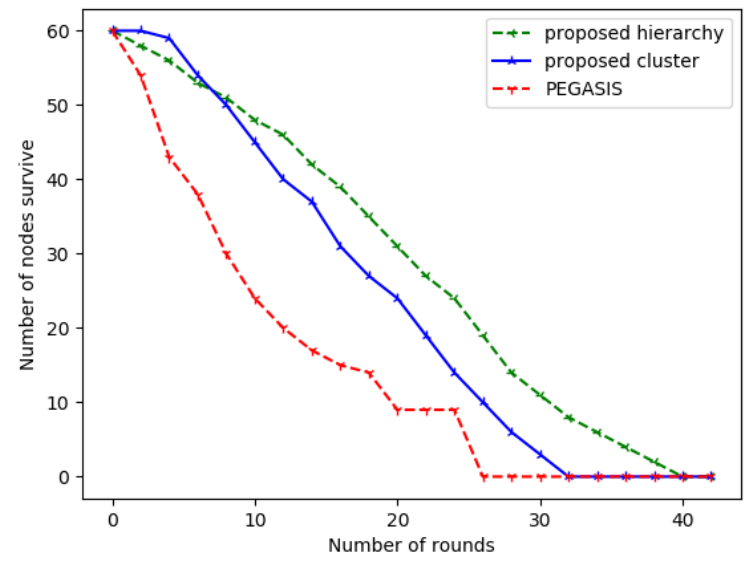

Figure 10. Comparison of network lifetime among the PEGASIS, proposed cluster and proposed hierarchy-based protocols

\section{ConClusions}

In this paper, we have proposed a new routing protocol for wireless sensor networks that is based on the two basic protocols, namely, LEACH and PEGASIS. LEACH is a clustering-based adaptive hierarchy-based protocol and PEGASIS is a chain-based protocol. There have been tremendous investigations to explore these two protocols since they are published. Many modified versions of these protocols are available in the research domain as well as in the field of application. Initially, we proposed the cluster-based algorithm and then we proposed here the hierarchy-based algorithm. This hierarchy-based algorithm outperforms our previously proposed cluster-based protocol and it is more energy-efficient.

In our simulation, we have considered that the sensor nodes are stationary for the duration of their lifetime and all of them are homogeneous, i.e. of the same type. We have only considered the lifetime of the network in our simulation since it is a very crucial parameter for a sensor network.

In the future we plan to consider a dynamic network with mobile sensors. Moreover, we plan to include other network factors such as network delay, bit error rate, data compression, and propagation delay of network. We also would like to explore on sensors that could survive at cryogenic to very high in aerospace applications.

\section{ACKNOWLEDGEMENTS}

The authors would like to thank NASA MSFC Faculty Fellowship program and their staff members for providing the opportunity to conduct this research. We also would like to thank engineers and scientists at NASA MSFC ES36 group who took time and shared their thoughts and ideas on using wireless sensor network technologies in space applications. The authors would like to express their sincere gratitude to the anonymous reviewers and the editorial board for their valuable comments and suggestions that improved the paper. 


\section{REFERENCES}

[1] M. Othmana and K. Shazalib, "Wireless sensor network applications: a study in environment monitoring system," International Symposium on Robotics and Intelligent Sensors 2012 (IRIS 2012), Procedia Engineering 41 (2012), pp. 1204-1210.

[2] K. Bouabdellaha, H. Noureddine, and S. Larbi, "Using wireless sensor networks for reliable forest fires detection," The 3rd International Conference on Sustainable Energy Information Technology (SEIT 2013), Procedia Computer Science 19 (2013), pp. 794-801.

[3] I. Akyildiz, W. Su, Y. Sankarasubramaniam, and E. Cayirci, "Wireless sensor networks: a survey," Computer Networks 38 (2002) 393-422.

[4] K. Akkaya and M. Younis, "A survey on routing protocols for wireless sensor networks," Ad Hoc Networks 3 (2005) 325-349.

[5] K. Romer and F. Mattern, "The Design Space of Wireless Sensor Networks," IEEE Wireless Communications, vol.11, issue 6, Dec. 2004.

[6] S. Sin, M. Woo, and C.Raghavendra, "Power-aware routing in mobile ad hoc networks," In proceedings ACM/lEEE Mobicom'98,1998.

[7] M. Zorzi and R. Rao, "Energy Management in Wireless Communications," In Proceedings $6^{\text {th }}$ WINLAB Workshop on Third Generation Wireless Information Network, Mar. 1997.

[8] O. Younis and S. Fahmy, "HEED: A Hybrid, Energy-Efficient, Distributed Clustering Approach for Ad-hoc Sensor Networks," IEEE Transactions on Mobile Computing, vol. 3 , issue 4, Oct. 2004.

[9] M. A. Mirza and R. M. Garimella, "PASCAL: Power aware sectoring based clustering algorithm for Wireless Sensor Networks," 2009 International Conference on Information Networking, Chiang Mai, India, Jan. 2009.

[10] J. Huang, D. Ruan, and W. Meng, "An annulus sector grid aided energy-efficient multi-hop routing protocol for wireless sensor networks," Computer Networks, vol. 147, pp. 38-48, Dec. 2018.

[11] S. Arjunan and P. Sujatha, "Lifetime maximization of wireless sensor network using fuzzy based unequal clustering and ACO based routing hybrid protocol,” Applied Intelligence, vol. 48, issue 8, Aug. 2018.

[12] K. A. Darabkh, M. Z. El-Yabroudi, and A. H.El-Mousa, "BPA-CRP: A balanced power-aware clustering and routing protocol for wireless sensor networks," Ad Hoc Networks, vol. 82, pp. 155171, Jan. 2019.

[13] M. Salam, K. Varnavas, and B. Maddireddy, "Prolonging wireless sensor network lifetime using routing protocol," in Proceedings of the $6^{\text {th }}$ IEEE International Conference on Wireless for Space and Extreme Environments (WiSEE), Dec. 11-13, Huntsville, Alabama, USA.

[14] W. Heinzelman, A. Chandrakasan, and H. Balakrishnan, "Energy-efficient communication protocol for wireless microsensor networks," in the Proceedings of the Hawaii International Conference on System Sciences, Jan. 4-7, 2000, Maui, Hawaii.

[15] W. Heinzelman, A. Chandrakasan, and H. Balakrishnan, "An application-specific protocol architecture for wireless microsensor networks," IEEE Trans. On Wireless Communications, vol. 1, no. 4, Oct. 2002.

[16] F. Xiangning and S. Yulin, "Improvement on LEACH protocol of wireless sensor network," in the Proceeding of the 2007 International Conference on Sensor Technologies and Applications (SENSORCOMM 2007), Valencia, Spain.

[17] M. Tong and M. Tang, "LEACH-B: An improved LEACH protocol for wireless sensor network," in the Proceeding of the $6^{\text {th }}$ IEEE International Conference on Wireless Communications Networking and Mobile Computing (WiCOM), Chengdu, China, Sep. 2010.

[18] H. Shwe, A. Kumar, and P. Chong, "Building efficient multi-level wireless sensor networks with cluster-based routing protocol," KSII Transaction on Internet ad Information Systems, vol. 10, no. 9, pp. 4272-4286, Sep. 2016.

[19] A. Al-Baz and A. El-Sayed, "A new algorithm for cluster head selection in LEACH protocol for wireless sensor networks," International Journal of Communication Systems, volume 31, issue 1, Sept. 2017.

[20] S. Lindsey and C. Raghavendra, "PEGASIS: power efficient gathering in sensor information systems," in the Proceedings of the IEEE Aerospace Conference, Big Sky, Montana, Mar. 2002.

[21] T. Li, F. Ruan, Z. Fan, J. Wang, and J. Kim, "An Improved PEGASIS Protocol for Wireless Sensor Network," in the IEEE Proceeding of the $20153^{\text {rd }}$ International Conference on Computer and Computing Science (COMCOMS), Hanoi, Vietnam. 
International Journal of Wireless \& Mobile Networks (IJWMN) Vol. 12, No. 3, June 2020

[22] S. Feng, B. Qi, and L. Tang, “An improved energy-efficient PEGASIS-based protocol in wireless sensor networks,", in the Proceeding of the IEEE $8^{\text {th }}$ International Conference on Fuzzy Systems and Knowledge

Discovery

(FSKD),

Shanghai,

China,

July

2011.

\section{AuTHORS}

Mohammad Abdus Salam received his bachelor's degree in electrical and electronics engineering from Rajshahi University of Engineering and Technology, Bangladesh in 1991. His master's and Ph.D. degrees are from the University of Fukui, Japan in 1998, 2001, respectively. He is currently a professor of Computer Science at Southern University, Baton Rouge, Louisiana. He has over 15 years of teaching and research experience. His research interest includes wireless sensor networks, wireless communication, and information and coding theory. He has taught a variety of courses from the field of computer science and engineering. He has authored and co-authored many international journals, conferences proceedings, and a book chapter. He served as a guest editor for many journals. He also served on the editorial boards and panelist for NSF, NASA, and many conferences.

He is a senior member of IEEE and an executive council member of Louisiana Academy of Sciences. He was awarded numerous awards throughout his career including the faculty outstanding achievement award from the President of the Southern University System and NASA faculty fellowship award.

Kosta Varnavas received his Bachelor of Science degree in electrical engineering from Christian Brothers College in 1988. Post graduate work has been done at the University of Alabama in Hunts ville.

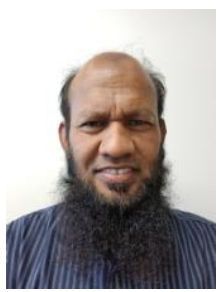

Varnavas has been working for NASA Marshall Space Flight Center since 1989 in a variety of digital design tasks. His current research areas wireless sensors and sensor design. His areas of concentration are the new sensor designs to allow complete control and flexibility from over the air networks.

Bhargava Reddy Maddireddy received his bachelor's degree in electrical and electronics engineering from Jawaharlal Nehru Technological University in 2010. His master's degree is from the Southern University and A\&M College, Baton Rouge, Louisiana in 2015.

$\mathrm{He}$ is currently a Network Security Engineer for the State of South Carolina. Maddireddy has more than 8 years of experience in IT attestation (SOC, HIPAA), risk management, compliance and privacy. Bhargava's primary focus is on emerging technology issues and privacy concerns for organizations. Bhargava is an active writer, speaker, and enjoys spending his time educating people on security and privacy.

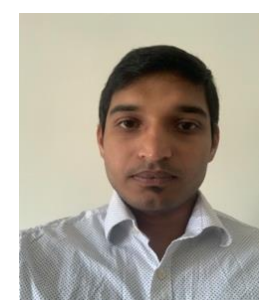

